\title{
Transition from Propagating Polariton Solitons to a Standing Wave Condensate Induced by Interactions
}

\author{
M. Sich, ${ }^{1, *}$ J. K. Chana, ${ }^{1,2}$ O. A. Egorov, ${ }^{3}$ H. Sigurdsson, ${ }^{4}$ I. A. Shelykh, ${ }^{4,5}$ D. V. Skryabin,,${ }^{6,5}$ P. M. Walker, ${ }^{1}$ \\ E. Clarke, ${ }^{7}$ B. Royall, ${ }^{1}$ M. S. Skolnick, ${ }^{1,5}$ and D. N. Krizhanovskii ${ }^{1,5, \dagger}$ \\ ${ }^{1}$ Department of Physics and Astronomy, The University of Sheffield, Sheffield S3 7RH, United Kingdom \\ ${ }^{2}$ Base4 Innovation, Ltd., Cambridge CB3 OFA, United Kingdom \\ ${ }^{3}$ Technische Physik der Universität Würzburg, Am Hubland 97074, Würzburg, Germany \\ ${ }^{4}$ Science Institute, University of Iceland, Dunhagi-3, IS-107 Reykjavik, Iceland \\ ${ }^{5}$ Department of Nanophotonics and Metamaterials, ITMO University, St. Petersburg 197101, Russia \\ ${ }^{6}$ Department of Physics, University of Bath, Bath BA2 7AY, United Kingdom \\ ${ }^{7}$ EPSRC National Centre for III-V Technologies, The University of Sheffield, Sheffield S1 4DE, United Kingdom
}

(Received 7 December 2017; published 19 April 2018)

\begin{abstract}
We explore phase transitions of polariton wave packets, first, to a soliton and then to a standing wave polariton condensate in a multimode microwire system, mediated by nonlinear polariton interactions. At low excitation density, we observe ballistic propagation of the multimode polariton wave packets arising from the interference between different transverse modes. With increasing excitation density, the wave packets transform into single-mode bright solitons due to effects of both intermodal and intramodal polariton-polariton scattering. Further increase of the excitation density increases thermalization speed, leading to relaxation of the polariton density from a solitonic spectrum distribution in momentum space down to low momenta, with the resultant formation of a nonequilibrium condensate manifested by a standing wave pattern across the whole sample.
\end{abstract}

DOI: $10.1103 /$ PhysRevLett.120.167402

Introduction.-Self-organization of nonlinear waves plays a fundamental role in a wide variety of phenomena, which in many cases has shaped the development of key areas of modern physics. These effects include BoseEinstein condensation (BEC) [1], spontaneous pattern formation [2], turbulence, solitons [3], and topological defects. Solitons are self-sustained objects characterized by energy localization in space and time through a balance between nonlinearity and dispersion. They contain a broad spectrum of waves with different energies and momenta. By contrast, $\mathrm{BEC}$ is characterized by a quasihomogeneous density distribution in real space and a narrow spectrum in momentum space. Both have been observed in cold atomic gases [4,5] and polariton systems [6].

In nonlinear optics, the interplay between nonlinearity, spatial, and temporal degrees of freedom is particularly interesting. It enables the study of ultrabroadband emission and multimode solitons [7] in fibers and BEC-like condensation of classical waves in nonlinear crystals [8]. Both effects arise from scattering between different transverse modes [7,9]. Describing such complex systems analytically or numerically poses great challenges. Kinetic wave theory and principles of thermodynamics $[10,11]$ have been used to explain supercontinuum generation in optical fibers [12], incoherent spectral solitons [13,14], and polariton condensation [15], while coupled nonlinear Schrödinger equations, which neglect any incoherent wave population, have been used to describe multimode solitons $[16,17]$.
Polaritons in optical microresonators, where strong exciton-photon hybridization enables giant $\chi^{(3)}$ optical nonlinearity $[18,19]$, form a unique laboratory for the study of nonlinear collective phenomena, including BEC and polariton lasing [20-23], self-organization through multiple polariton-polariton scattering [24], quantized vortices [25,26], and solitons [27-29]. While in planar 2D microcavities polariton-polariton scattering usually occurs between the states residing in a single band formed by the lower polariton branch [30], a range of scattering channels opens up in laterally confined systems, such as microcavity wires (MCWs) [31,32], where nonlinear interactions can mix between different transverse polariton modes [33]. Theoretically, this mixing can lead to competition between modes of different parity and formation of parity switching waves and parity solitons under static nonresonant excitation $[34,35]$.

In this Letter, we demonstrate, both experimentally and numerically, an evolution of a multimode polariton system between phases of contrasting properties: multimode wave packet $\rightarrow$ soliton $\rightarrow$ soliton doublet $\rightarrow$ dynamic condensate. The crucial ingredients for the observed evolution are the specific shape of the microcavity polariton dispersion, arising from strong exciton-photon coupling, where the sign of the polariton effective mass changes from positive to negative with increasing momentum, the existence of multiple transverse modes, and strong polariton nonlinearities. In 
the nonlinear regime, polariton-polariton interactions redistribute the particles between several transverse lower polariton modes. At intermediate powers, propagating bright single- and double-peak solitons form, which are characterized by a dominant occupation in a finite range of nonzero momenta just above the point of inflexion of the dispersion curve. At even stronger excitation, cascading polaritonpolariton and polariton-exciton scattering leads to relaxation of the polariton density from the solitonic mode to lower momenta, and a nonequilibrium analog of BEC is formed, characterized by a standing wave pattern. It is possible to achieve this quasithermalized state because the long polariton lifetime of $\simeq 30$ ps and very strong Kerr-like polariton nonlinearity (leading to interaction times much shorter then the lifetime) allow efficient redistribution of polariton density. These observations are realized in a $100 \mu \mathrm{m}$-long MCW.

To compare the experimental results with theoretically expected behavior, we used the generalized GrossPitaevskii equation (see, e.g., [36]), with an additional phenomenological nonradiative excitonic decay accounting for decoherence. Details of the modeling are given in the Supplemental Material [37]. Previously, conservative bright polariton solitons have been reported in a narrow and long MCW [41], where only the ground polariton transverse mode was excited and multimode evolution, mode competition, or standing wave condensation were not observed. In multimode polariton systems, condensation [31,32] and ballistic propagation [42] have only been reported separately. In optical fibers, where typically solitons and supercontinuum generation are observed (see, e.g., [43]), spectral narrowing [44] and spectral condensation in ultralong fiber lasers [45] were reported, but again a transition from soliton to condensate behavior was not observed.

Results.-Our sample is a $3 \lambda / 2$ microcavity with 3 InGaAs quantum wells (10 nm thick, $4 \%$ indium) and was previously described in Ref. [46]. Distributed Bragg mirrors are $\mathrm{GaAs} / \mathrm{AlGaAs}(85 \% \mathrm{Al})$ with 26 (23) repeats on the bottom (top) mirror. The Rabi splitting and polariton lifetime are $\simeq 4.12 \mathrm{meV}$ and $\simeq 30$ ps. The top mirror was partially etched defining $100 \mu \mathrm{m}$-long, $8 \mu \mathrm{m}$-wide mesas [Fig. 1(a)]. The lateral confinement of the photonic mode generates discrete energy levels labeled as $n=0,1,2, \ldots$. (where $n$ is the number of nodes in the photon field distribution across the wire), which can be seen in the far-field polariton photoluminescence under a low-power nonresonant excitation [Fig. 1(b)]. The ground, $n=0$, photonic mode is detuned by $\simeq-4.07 \mathrm{meV}$ from the exciton at $1490 \mathrm{meV}$.

We applied a quasiresonant pulsed excitation laser at an angle of incidence relative to the sample top surface corresponding to $k_{x} \simeq 2.4 \mu \mathrm{m}^{-1}$ and $k_{y} \simeq 0$. The excitation beam was spectrally filtered to approximately 5-7 ps duration FWHM (corresponding to $\simeq 0.3 \mathrm{meV}$ energy width) and focused into a spot size of $\simeq 20 \mu \mathrm{m}$ close to (a)
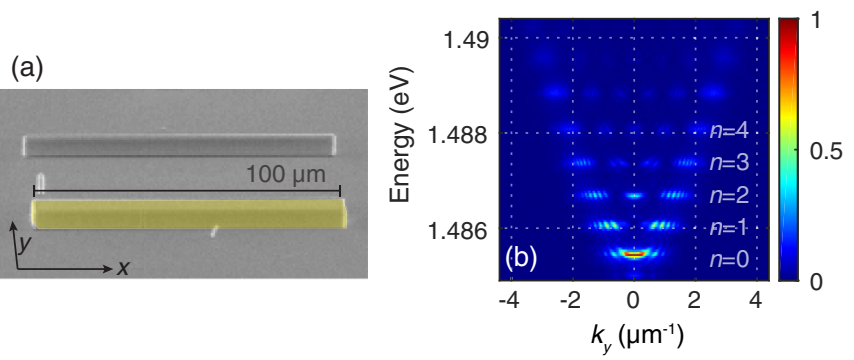

FIG. 1. (a) SEM image of the sample with etched microwires. The $8 \times 100 \mu \mathrm{m}$ wire is shaded in yellow. (b) Energy-momentum dispersion of the lower polariton branch measured across the wire, along the $y$ axis, showing different energy modes arising from lateral photonic confinement. The fine modulation of the mode dispersions arises from interference due to reflection from the polished side of the substrate [47].

one end of the wire. The finite width of the pulse in momentum, $\Delta k_{x} \simeq 0.4 \mu \mathrm{m}^{-1}$, as well as Rayleigh scattering from the edges of the etched MCW enables efficient excitation of three $(n=0,1,2)$ transverse lower polariton modes [Fig. 2(e)]. We start with the lowest excitation power, $P_{1}=90 \mu \mathrm{W}$ (Fig. 2), corresponding to $P_{0}=1 \mathrm{meV} \mu \mathrm{m}^{-2}$ in the numerical modeling [37], when polariton-polariton interactions are negligible. The excited polariton modes have different group velocities in the range of $\sim 1-3 \mu \mathrm{m} / \mathrm{ps}$, which, in addition to polariton group velocity dispersion (GVD) of each transverse polariton mode, leads to spreading of the pulse in real space. The interference
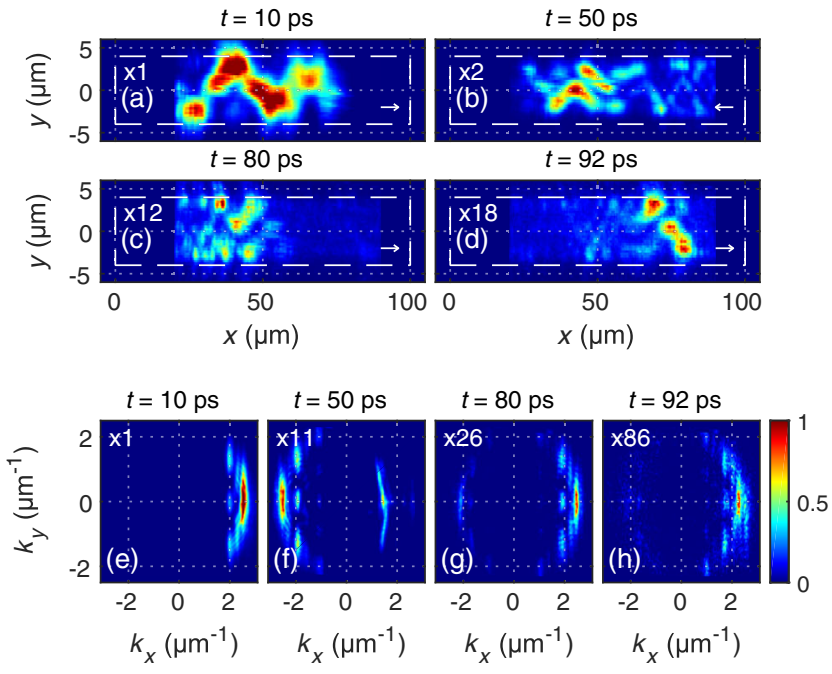

FIG. 2. Low-power, $P_{1}=90 \mu \mathrm{W}$, emission characterization. (a)-(d) Reconstructed real-space images of the polariton pulse propagating in the MCW at different times. White dashed rectangles show the outline of the MCW. Arrows in lower right corners indicate the direction of travel of the pulses. (e)-(h) The corresponding snapshots of the momentum space at the same times as (a)-(d), respectively. All pseudocolor scales are linear zero to one, and numbers in top right corners of each panel are intensity scaling factors applied to data for each panel. 
between the transverse modes also results in a visible "snaking" (see Ref. [42]) of the pulse in real space [Fig. 2(a) and in the theory Figs. S3(a)-S3(c)] with frequency $\omega_{s}=\hbar\left(k_{x, n=0}^{2}-k_{x, n=1}^{2}\right) / 2 m$; see Fig. S4.

The long polariton lifetime allows us to observe several cycles of the pulse moving back and forth along the wire. Figure 2 provides several snapshots of this process, showing the real-space images and the corresponding $k$-space distributions. Within $\simeq 30$ ps after the excitation, the front of the pulse quickly reaches the end of the wire, where it is elastically reflected backwards so that the momentum of polariton emission changes its sign [Fig. 2(f)] [48]. The same is seen in the modeling in Figs. S2(f) and S2(j). During reflections from the ends of the wire, polariton modes of higher orders, i.e., $n=3$ and 4 , are also populated through the elastic scattering of the pulse from imperfections [Figs. 2(f)-2(h)]. The interference between low- and high-order modes enhances the overall pulse spreading and produces more complex real-space patterns [Figs. 2(b)-2(d)]. Overall, at the low pump power, the momentum emission associated with different modes is almost the same at $\simeq 10$ and $\simeq 90 \mathrm{ps,} \mathrm{confirming}$ low efficiency of polariton relaxation in energy-momentum space due to weak interactions with phonons, which is also reproduced in our modeling (see Fig. S3 of the Supplemental Material [37]).

At intermediate power, $P_{2}=540 \mu \mathrm{W}$, corresponding to $P_{0}=5 \mathrm{meV} \mu \mathrm{m}^{-2}$ in the numerical modeling [37], the excitation $k$ vector plays a crucial role. Namely, since the point of inflexion of the lower polariton mode $(n=0)$ is at $\simeq 2.1 \mu \mathrm{m}^{-1}$, polaritons excited by the pump in $n=0$ have a negative effective mass. Hence, the interplay of the polariton GVD with the repulsive interactions can enable soliton formation [28]. Snapshots of pulse evolution in real and momentum space are shown in Fig 3. The initial pulse
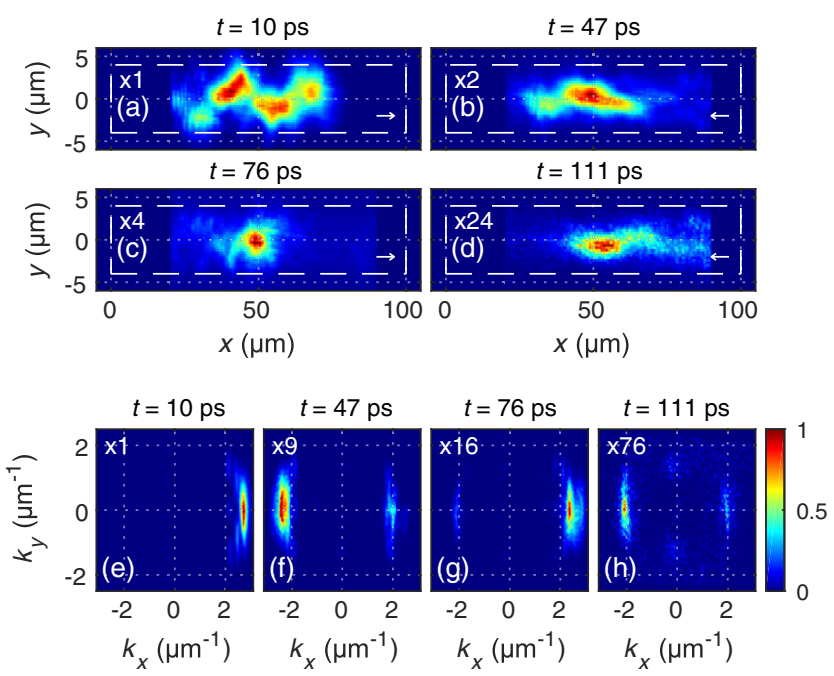

FIG. 3. Medium-power, $P_{2}=540 \mu \mathrm{W}$, emission characterization for different times. Description of (a)-(h) is the same as in Fig. 2. propagation is very similar to the case of the low power $P_{1}$, as can be seen by comparing panels (a) and (b) in Figs. 3 and 2. However, in contrast to the low-power behavior, here, at later times (50-80 ps), the polariton nonlinearity results in the emergence of a single dominant mode, when individual energy levels can no longer be resolved in the momentum space [Figs. 3(f) and 3(g), also Figs. S4(c)-S4(g), S4(j), and S4(k) of the Supplemental Material [37] ], which coincides with a significant narrowing of the pulse in real space (and hence in time) down to $\simeq 10 \mu \mathrm{m}$, as in Fig. 3(c). The ratio between the peak intensities at 50$80 \mathrm{ps}$ and $10 \mathrm{ps}$ [Figs. $3(\mathrm{f})-3(\mathrm{~h})]$ is $\simeq 1.6$ times higher than the same ratio at low power [Figs. 2(f)-2(h)]. This is consistent with the concentration of pulse energy in the ground mode.

Kerr-like nonlinear interactions between transverse photonic modes in nonlinear crystals and optical fibers have been shown to lead to emergence of solitons and condensation of classical waves $[8,11]$. A similar process occurs in the polariton $\mathrm{MCW}$, where polaritons, excited within a certain momentum (energy) range, populate other initially empty polariton states through nonlinear polaritonpolariton scattering. In turn, this maximizes the population of the ground mode $n=0$ in the range of high momenta $\left(k \sim 2-2.5 \mu \mathrm{m}^{-1}\right)$. The interplay between negative polariton mass and nonlinear repulsive interactions between polaritons with different momenta in the ground mode leads to self-focusing and evolution of the system towards a temporal soliton at 50-75 ps. Some of the corresponding scattering channels are depicted in Fig. 4(a): interactions between polaritons residing initially in modes $n=1$ and 2 result in a drastic increase of occupation in mode $n=0$ as well as the occupation of higher order modes $(n=3,4$, and 5). Furthermore, both intermodal and intramodal scattering
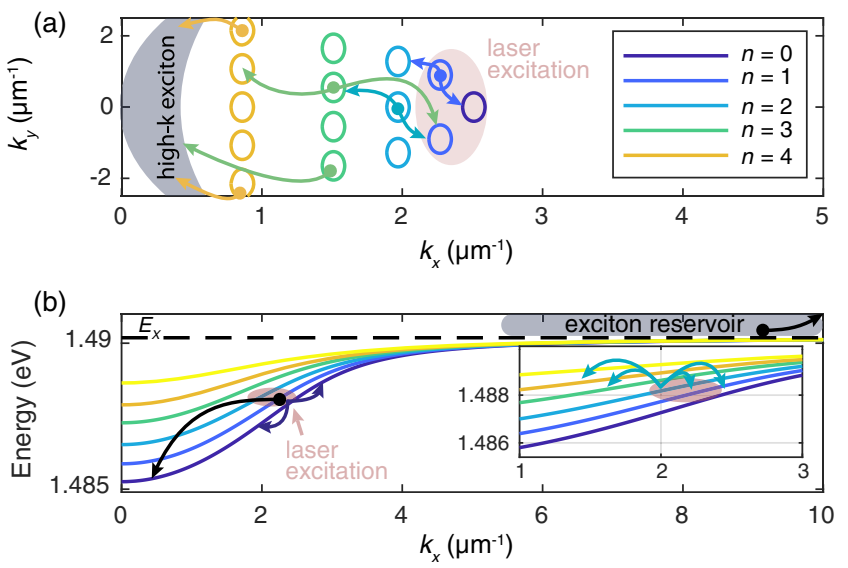

FIG. 4. (a) Schematic of some of the possible combinations for intermodal polariton scattering in $k$ space at a fixed energy $(\simeq 1.488 \mathrm{eV})$; the colored ovals approximate location and width of different lower polariton energy modes. (b) Schematic of intramodal polariton-polariton and polariton-exciton scattering leading to polariton relaxation. The inset corresponds to intermodal scattering shown in (a). 
spreads the polariton population over a large range of $k$ vectors, thus minimizing peak intensities in momentum space of the excited transverse $(n \geq 1)$ modes relative to the solitonic emission at $n=0$. Note that the polariton population (and hence nonlinearity) diminishes with time due to the finite lifetime, which together with the GVD leads to broadening of the wave packet at later times ( $>75 \mathrm{ps}$ ). The experimental results in Figs. 3(f)-3(h) are reproduced by the numerical modeling only when we include coherent interactions between multiple transverse modes (see Fig. S4 of the Supplemental Material [37]).

The soliton regime described above does not correspond to a thermalized state, which is not achievable at the intermediate excitation power due to the finite polariton lifetime. However, at a higher excitation power, thermalization can speed up due to the increased rate of polaritonpolariton scattering. At $P_{3}=800 \mu \mathrm{W}$, corresponding to $P_{0}=7 \mathrm{meV} \mu \mathrm{m}^{-2}$ in the numerical modeling [37], a soliton doublet [29], corresponding to the soliton fission regime, emerges already at $10-15$ ps after the excitation and remains stable until $\simeq 75$ ps [Figs. 5(b) and 5(c)]. By $30-40 \mathrm{ps}$, the emission follows the $k_{y}$ profile of the ground mode, with a single antinode, and has the broad modulated spectrum arising from broadband inter- and intramodal polariton-polariton scattering (modulation instability), as can be seen in Figs. 5(h) and 5(i). A large part of the soliton
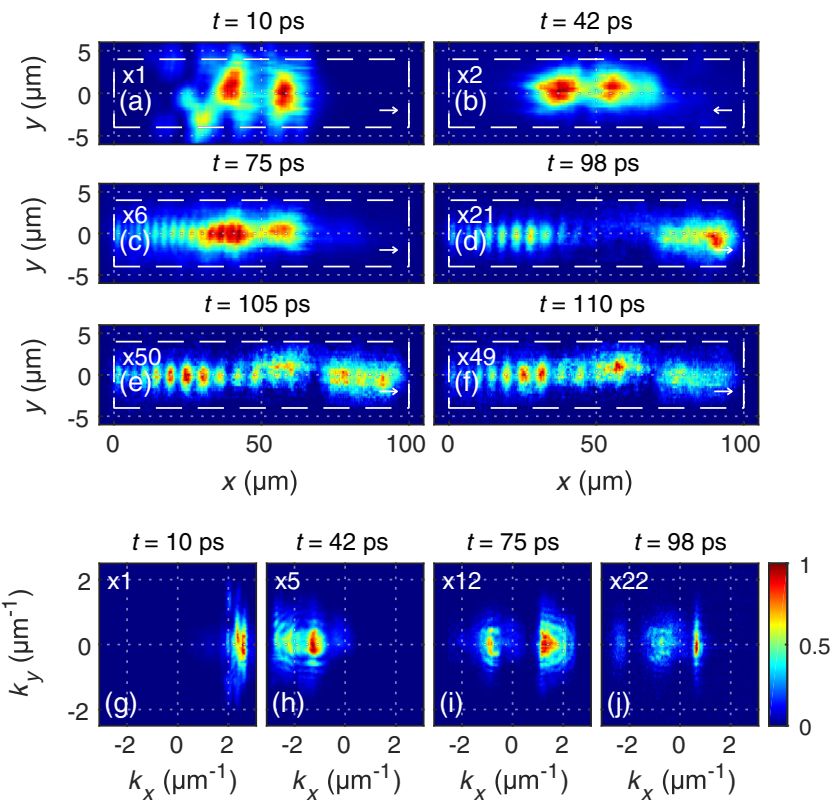

FIG. 5. High-power, $P_{3}=800 \mu \mathrm{W}$, emission characterization for different times. (a)-(f) Reconstructed real-space images of the polariton pulse propagating in the $\mathrm{MCW}$ at different times. White dashed rectangles show the outline of the $\mathrm{MCW}$. Arrows in lower right corners indicate the direction of travel of the pulses. (g)-(j) Corresponding snapshots of the momentum space at the same times as (a)-(d), respectively. All pseudocolor scales are linear zero to one, and numbers in top right corners of each panel are intensity scaling factors applied to data for each panel. doublet spectrum now lies below the point of inflexion (at $k_{x} \simeq 1.8 \mu \mathrm{m}^{-1}$ ), in the region where polariton effective mass is positive and where wave packet defocusing is expected. In this case, solitons can give up their energy to extended dispersive modes with lower $k$ vectors via Cherenkov radiation [41,49,50]. From a microscopical point of view, this process again can be understood as a result of multiple polariton-polariton scattering events. During each of these, a pair of polaritons of the same energy scatter one to a lower and the second to a higher energy state. This mechanism results in a gradual shift of the maximum of the polariton distribution to lower $k$ vectors since the high-energy, high- $k$ polaritons experience greater losses due to scattering to high-density, highmomenta excitonlike states, the so-called exciton reservoir [51]. The losses may arise from interaction of polaritons with excitonic disorder [52,53] or polariton-phonon and polariton-electron scattering [54]. The mechanisms involving the reservoir are not directly taken into account in our numerical modeling (which reproduces experimental results well, see Fig. S5 of the Supplemental Material [37]), but are accounted for phenomenologically by introducing excitonic decay rates higher than photonic. Furthermore, even though the energy of the lower polariton states is below that of bare uncoupled excitons, coherent pair polariton-polariton scattering may also effectively populate the latter mainly due to a very high density of exciton states [Fig. 4(a)]. This is confirmed by our simulations [see Figs. S5(j)-S5(l) of the Supplemental Material [37]]. Finally, note that polariton scattering with high-momenta excitons shown in Fig. 4(b) probably also plays an important role in the polariton relaxation [55]. Therefore, a number of mechanisms are potentially involved in spectral redistribution of polaritons in the wave packet.

At $\simeq 75 \mathrm{ps}$, slow counterpropagating waves emerge in the wire at $k_{x} \simeq \pm 0.8-1 \mu \mathrm{m}^{-1}$ leading to formation of a modulated tail behind the doublet. At $\simeq 100 \mathrm{ps,} \mathrm{the} \mathrm{polar-}$ iton emission mostly peaks at $k_{x} \simeq 0.5-0.7 \mu \mathrm{m}^{-1}$, lower than the momentum of the excitation pulse. This corresponds to onset of a standing wave with 17 maxima seen in Figs. 5(d)-5(f). The same effect is also observed in our modeling in Figs. S5(d), S5(h), and S5(j) of the Supplemental Material [37]. This standing wave arises from the interference across the whole wire between two waves at $k_{x} \simeq \pm 0.5-0.6 \mu \mathrm{m}^{-1}$, which are long-range and coherent and hence form a macroscopically occupied state (a dynamic analog of a nonequilibrium BEC).

Discussion.-Our findings show that, in a sample with a long polariton lifetime, condensates can emerge out of a resonantly excited polariton cloud. By varying the energy, bandwidth, and power of the excitation pulse, it is possible to control the excited polariton modes and their energymomentum distributions. Resonant excitation can also allow control of the spin degree of freedom, which can 
be useful for investigation of Berezinskii-KosterlitzThouless phases [56] associated with the emergence of half- or full-spin vortex excitations in polariton systems with spin-anisotropic interactions [57], so far a completely unexplored field.

All our experimental observations are qualitatively reproduced by our numerical modeling. This shows that the condensation arises fundamentally from the very strong nonlinear response in the generalized Gross-Pitaevskii equation (GPE) used to describe the polariton system. While we employ direct numerical integration, wave turbulence theories have been applied to a wide variety of GPE-type systems to explain classical condensation as irreversible evolution to a thermodynamic equilibrium state [10]. In 3D, condensation to either soliton [58] or cw [11] states has been studied for focusing or defocusing conditions, respectively. Condensation was also shown theoretically for nonlocal and saturable nonlinearities and defocusing 2D and multimode waveguide systems [10]. The microcavity polariton dispersion provides a transition from focusing to defocusing as the mass changes sign and we observe the transition from solitonic to condensate final state with increasing density, even though the pump always corresponds to focusing conditions. An interesting experimental and theoretical perspective would be to study the equilibrium state of systems with this dispersion. We note also that one can extend theories to account for the couplings to phonon and exciton reservoirs present in polariton systems using kinetic Boltzmann [59] or stochastic GPE [60] approaches.

M.S. and D. N.K. acknowledge support from the Leverhulme Trust Grant No. RPG-2013-339. M. S., J. K. C., P.M.W., B. R., M.S.S., and D.N.K. acknowledge the support from the EPSRC Grants No. EP/J007544/1 and No. EP/N031776/1 and the ERC Advanced Grant EXCIPOL 320570. D. V.S. acknowledges the Russian Foundation for Basic Research (16-52-150006) and the ITMO University Fellowship through the Government of Russia Grant No. 074-U01. H. S. and I. A. S. acknowledge the support by the Research Fund of the University of Iceland, The Icelandic Research Fund, Grant No. 163082-051 and the Project 3.2614.2017/4.6 of the Ministry of Education and Science of Russian Federation. I. A. S., M. S. S., and D. N. K. Megagrant No. 14.Y26.31.0015 of the Ministry of Education and Science of Russian Federation. We thank Marzena Szymańska for helpful discussions.

*m.sich@sheffield.ac.uk

†.krizhanovskii@sheffield.ac.uk

[1] Universal Themes of Bose-Einstein Condensation, edited by N. P. Proukakis, D. W. Snoke, and P. B. Littlewood (Cambridge University Press, Cambridge, England, 2017) p. 649 .
[2] C. E. Whittaker, B. Dzurnak, O. A. Egorov, G. Buonaiuto, P. M. Walker, E. Cancellieri, D. M. Whittaker, E. Clarke, S. S. Gavrilov, M. S. Skolnick, and D. N. Krizhanovskii, Phys. Rev. X 7, 031033 (2017).

[3] Y.S. Kivshar and G.P. Agrawal, Optical Solitons (Academic Press, New York, 2003), 1st ed..

[4] L. Khaykovich, F. Schreck, G. Ferrari, T. Bourdel, J. Cubizolles, L. D. Carr, Y. Castin, and C. Salomon, Science 296, 1290 (2002).

[5] K. E. Strecker, G. B. Partridge, A. G. Truscott, and R. G. Hulet, Nature (London) 417, 150 (2002).

[6] M. Sich, D. V. Skryabin, and D. N. Krizhanovskii, C.R. Phys. 17, 908 (2016).

[7] L. G. Wright, S. Wabnitz, D. N. Christodoulides, and F. W. Wise, Phys. Rev. Lett. 115, 223902 (2015).

[8] C. Sun, S. Jia, C. Barsi, S. Rica, A. Picozzi, and J. W. Fleischer, Nat. Phys. 8, 470 (2012).

[9] K. Krupa, A. Tonello, A. Barthélémy, V. Couderc, B. M. Shalaby, A. Bendahmane, G. Millot, and S. Wabnitz, Phys. Rev. Lett. 116, 183901 (2016).

[10] A. Picozzi, J. Garnier, T. Hansson, P. Suret, S. Randoux, G. Millot, and D. N. Christodoulides, Phys. Rep. 542, 1 (2014).

[11] C. Connaughton, C. Josserand, A. Picozzi, Y. Pomeau, and S. Rica, Phys. Rev. Lett. 95, 263901 (2005).

[12] B. Barviau, B. Kibler, A. Kudlinski, A. Mussot, G. Millot, and A. Picozzi, Opt. Express 17, 7392 (2009).

[13] A. Picozzi, S. Pitois, and G. Millot, Phys. Rev. Lett. 101, 093901 (2008).

[14] A. V. Gorbach and D. V. Skryabin, Opt. Lett. 31, 3309 (2006).

[15] D. Snoke, G. Liu, and S. Girvin, Ann. Phys. (Amsterdam) 327, 1825 (2012).

[16] F. Poletti and P. Horak, J. Opt. Soc. Am. B 25, 1645 (2008).

[17] A. A. Sukhorukov, A. Ankiewicz, and N. N. Akhmediev, Opt. Commun. 195, 293 (2001).

[18] N. A. Gippius, I. A. Shelykh, D. D. Solnyshkov, S. S. Gavrilov, Y. G. Rubo, A. V. Kavokin, S. G. Tikhodeev, and G. Malpuech, Phys. Rev. Lett. 98, 236401 (2007).

[19] P. M. Walker, L. Tinkler, D. V. Skryabin, A. Yulin, B. Royall, I. Farrer, D. A. Ritchie, M. S. Skolnick, and D. N. Krizhanovskii, Nat. Commun. 6, 8317 (2015).

[20] J. Kasprzak, M. Richard, S. Kundermann, A. Baas, P. Jeambrun, J. M. J. Keeling, F. M. Marchetti, M. H. Szymańska, R. André, J. L. Staehli, V. Savona, P. B. Littlewood, B. Deveaud, and L. S. Dang, Nature (London) 443, 409 (2006).

[21] M. Galbiati, L. Ferrier, D. D. Solnyshkov, D. Tanese, E. Wertz, A. Amo, M. Abbarchi, P. Senellart, I. Sagnes, A. Lemaître, E. Galopin, G. Malpuech, and J. Bloch, Phys. Rev. Lett. 108, 126403 (2012).

[22] D. Bajoni, P. Senellart, E. Wertz, I. Sagnes, A. Miard, A. Lemaître, and J. Bloch, Phys. Rev. Lett. 100, 047401 (2008).

[23] Y. Sun, P. Wen, Y. Yoon, G. Liu, M. Steger, L. N. Pfeiffer, K. West, D. W. Snoke, and K. A. Nelson, Phys. Rev. Lett. 118, 016602 (2017).

[24] D. N. Krizhanovskii, S. S. Gavrilov, A. P. D. Love, D. Sanvitto, N. A. Gippius, S. G. Tikhodeev, V. D. Kulakovskii, D. M. Whittaker, M. S. Skolnick, and J. S. Roberts, Phys. Rev. B 77, 115336 (2008). 
[25] K. G. Lagoudakis, M. Wouters, M. Richard, A. Baas, I. Carusotto, R. Andre, L. S. Dang, and B. Deveaud-Pledran, Nat. Phys. 4, 706 (2008).

[26] G. Tosi, G. Christmann, N. G. Berloff, P. Tsotsis, T. Gao, Z. Hatzopoulos, P. G. Savvidis, and J. J. Baumberg, Nat. Commun. 3, 1243 (2012).

[27] A. Amo, S. Pigeon, D. Sanvitto, V. G. Sala, R. Hivet, I. Carusotto, F. Pisanello, G. Leménager, R. Houdré, E. Giacobino, C. Ciuti, and A. Bramati, Science 332, 1167 (2011).

[28] M. Sich, D. N. Krizhanovskii, M. S. Skolnick, A. V. Gorbach, R. Hartley, D. V. Skryabin, E. A. Cerda-Méndez, K. Biermann, R. Hey, and P. V. Santos, Nat. Photonics 6, 50 (2012).

[29] J. K. Chana, M. Sich, F. Fras, A. V. Gorbach, D. V. Skryabin, E. Cancellieri, E. A. Cerda-Méndez, K. Biermann, R. Hey, P. V. Santos, M. S. Skolnick, and D. N. Krizhanovskii, Phys. Rev. Lett. 115, 256401 (2015).

[30] R. M. Stevenson, V. N. Astratov, M. S. Skolnick, D. M. Whittaker, M. Emam-Ismail, A. I. Tartakovskii, P. G. Savvidis, J. J. Baumberg, and J. S. Roberts, Phys. Rev. Lett. 85, 3680 (2000).

[31] L. Ferrier, E. Wertz, R. Johne, D. D. Solnyshkov, P. Senellart, I. Sagnes, A. Lemaître, G. Malpuech, and J. Bloch, Phys. Rev. Lett. 106, 126401 (2011).

[32] E. Wertz, L. Ferrier, D. D. Solnyshkov, R. Johne, D. Sanvitto, A. Lemaître, I. Sagnes, R. Grousson, A. V. Kavokin, P. Senellart, G. Malpuech, and J. Bloch, Nat. Phys. 6, 860 (2010).

[33] G. Dasbach, M. Schwab, M. Bayer, D. N. Krizhanovskii, and A. Forchel, Phys. Rev. B 66, 201201 (2002).

[34] H. Sigurdsson, I. A. Shelykh, and T. C. H. Liew, Phys. Rev. B 92, 195409 (2015).

[35] H. Sigurdsson, T. C. H. Liew, and I. A. Shelykh, Phys. Rev. B 96, 205406 (2017).

[36] I. Carusotto and C. Ciuti, Phys. Rev. Lett. 93, 166401 (2004).

[37] See Supplemental Material at http://link.aps.org/ supplemental/10.1103/PhysRevLett.120.167402 for details of numerical modeling of kinetic evolution of polariton wave packets, which includes Refs. [36,38-40].

[38] V. Savona, J. Phys. Condens. Matter 19, 295208 (2007).

[39] F. Baboux, L. Ge, T. Jacqmin, M. Biondi, E. Galopin, A. Lemaître, L. Le Gratiet, I. Sagnes, S. Schmidt, H. E. Türeci, A. Amo, and J. Bloch, Phys. Rev. Lett. 116, 066402 (2016).

[40] W. Pauli, Wave Mechanics, Pauli Lectures on Physics (Dover Publications, New York, 2000), Vol. 5, p. 240.

[41] D. V. Skryabin, Y. V. Kartashov, O. A. Egorov, M. Sich, J. K. Chana, L. E. Tapia Rodriguez, P. M. Walker, E. Clarke, B. Royall, M. S. Skolnick, and D. N. Krizhanovskii, Nat. Commun. 8, 1554 (2017).
[42] C. Antón, T. C. H. Liew, G. Tosi, M. D. Martín, T. Gao, Z. Hatzopoulos, P. S. Eldridge, P. G. Savvidis, and L. Viña, Phys. Rev. B 88, 035313 (2013).

[43] D. V. Skryabin and A. V. Gorbach, Rev. Mod. Phys. 82, 1287 (2010).

[44] S. K. Turitsyn, A. E. Bednyakova, M. P. Fedoruk, S. B. Papernyi, and W. R. Clements, Nat. Photonics 9, 608 (2015).

[45] E. G. Turitsyna, G. Falkovich, V. K. Mezentsev, and S. K. Turitsyn, Phys. Rev. A 80, 031804(R) (2009).

[46] L. Tinkler, P. M. Walker, E. Clarke, D. N. Krizhanovskii, F. Bastiman, M. Durska, and M. S. Skolnick, Appl. Phys. Lett. 106, 021109 (2015).

[47] A. I. Tartakovskii, D. N. Krizhanovskii, and V. D. Kulakovskii, Phys. Rev. B 62, R13298 (2000).

[48] Emission at small positive values of $k_{x}\left(\simeq+1.5 \mu \mathrm{m}^{-1}\right)$ relative to the magnitudes at $-k_{x}$ in Fig. 2(f) may be due to the effect of disorder scattering on reflection at the end of the MCW.

[49] K. Hammani, B. Kibler, C. Finot, and A. Picozzi, Phys. Lett. A 374, 3585 (2010).

[50] D. V. Skryabin, F. Luan, J. C. Knight, and P. S. J. Russell, Science 301, 1705 (2003).

[51] P. M. Walker, L. Tinkler, B. Royall, D. V. Skryabin, I. Farrer, D. A. Ritchie, M. S. Skolnick, and D. N. Krizhanovskii, Phys. Rev. Lett. 119, 097403 (2017).

[52] D. N. Krizhanovskii, A. I. Tartakovskii, A. V. Chernenko, V. D. Kulakovskii, M. Emam-Ismail, M. S. Skolnick, and J. S. Roberts, Solid State Commun. 118, 583 (2001).

[53] V. D. Kulakovskii, A. I. Tartakovskii, D. N. Krizhanovskii, M. S. Skolnick, and J.S. Roberts, Physica (Amsterdam) 13E, 455 (2002).

[54] A. I. Tartakovskii, D. N. Krizhanovskii, G. Malpuech, M. Emam-Ismail, A. V. Chernenko, A. V. Kavokin, V. D. Kulakovskii, M. S. Skolnick, and J. S. Roberts, Phys. Rev. B 67, 165302 (2003).

[55] P. G. Savvidis, J. J. Baumberg, D. Porras, D. M. Whittaker, M. S. Skolnick, and J. S. Roberts, Phys. Rev. B 65, 073309 (2002).

[56] D. Caputo, D. Ballarini, G. Dagvadorj, C. S. Muñoz, M. De Giorgi, L. Dominici, K. West, L. Pfeiffer, G. Gigli, F. P. Laussy, M. H. Szymańska, and D. Sanvitto, Nat. Mater. 17, 145 (2018).

[57] M. Vladimirova, S. Cronenberger, D. Scalbert, K. V. Kavokin, A. Miard, A. Lemaître, J. Bloch, D. Solnyshkov, G. Malpuech, and A. V. Kavokin, Phys. Rev. B 82, 075301 (2010).

[58] R. Jordan and C. Josserand, Phys. Rev. E 61, 1527 (2000).

[59] I. G. Savenko, T. C. H. Liew, and I. A. Shelykh, Phys. Rev. Lett. 110, 127402 (2013).

[60] M. Wouters, T. C. H. Liew, and V. Savona, Phys. Rev. B 82, 245315 (2010). 